are related to poor perception of health but not to pain sensitivity or cerebral processing of pain. Arthritis \& Rheumatology Journal 62(11).

[2] Ugar M, Sarp U, Karaaslan O, Tanik N, Arik HO (2015) Health anxiety and depression in patients with fibromyalgia syndrome. Journal of Inernational Medical Research 45(3):679-685.

Acknowledgements: NIL.

Disclosure of Interest: None declared

DOI: 10.1136/annrheumdis-2017-eular.1445

\section{SAT0730-HPR DIFFERENCES IN PERCEPTION OF THE DISEASE CONSTRAINTS BETWEEN THE CHILD AND PARENTS IN JIA}

E. Ünal ${ }^{1}$, P. Kısacık ${ }^{1}$, G. Arin ${ }^{1}$, N.B. Karaca ${ }^{1}$, E.D. Batu ${ }^{2}$, Z.S. Arıcı ${ }^{2}$, E. Sönmez ${ }^{2}$, Y. Bilginer ${ }^{2}$, S. Özen ${ }^{2} .{ }^{1}$ Department of Physiotherapy and Rehabilitation, Hacettepe University Faculty of Health Science; ${ }^{2}$ Pediatric Rheumatology, Hacettepe University Faculty of Medicine, Ankara, Turkey

Background: Studies suggest that the perception of the child and family differ in juvenile idiopathic arthritis. This may pose a problem for the clinician's assessment $(1,2)$

Objectives: The aim of this study was to evaluate the differences in perception of the disease constraints between child and parent in JIA.

Methods: 129 children with JIA were included in the study.The main complaint about the illness was requested to be expressed in writing by both the child and the family. Individuals' expressions and demographic data were recorded. The main complaints were considered nominal. The correlation between the parent's and child's expressions were examined. The types of complaints were classified as none, functional, symptomatic, and both functional and symptomatic.

Results: The age range of children ranged from 6 to 21 (mean $\pm S D ; 12,86 \pm 3,68$ ). When the complaints of the 129 children and the parents were compared, $45,7 \%$ were different, $20,2 \%$ were partially similar and $34,1 \%$ were perfectly similar complaints. When the answers of the 59 children and parents who differ in their complaints were examined; $67.8 \%$ of the children expressed a milder disease than their parents (Table1). While the family mostly concentrated on the symptoms, the children were worried on functional complaints (Table1).

Table 1. Results of the evaluations

\begin{tabular}{lcc}
\hline & $\begin{array}{c}\text { Child + Parent } \\
(\mathrm{n}=129)\end{array}$ & $\begin{array}{c}\text { Differing perception of problems } \\
\text { in the child and parent }(\mathrm{n}=59)\end{array}$ \\
\hline $\begin{array}{l}\text { Group of complaint - Child } \\
\text { None } \mathrm{n}(\%)\end{array}$ & $49(38)$ & $16(27,1)$ \\
$\quad$ Function $\mathrm{n}(\%)$ & $43(33,3)$ & $23(39)$ \\
$\quad$ Symptom n (\%) & $20(15,5)$ & $9(15,3)$ \\
$\quad$ Function + Symptom n (\%) & $17(13,2)$ & $11(18,6)$ \\
Group of complaint - Parent & & $16(27,1)$ \\
$\quad$ None $\mathrm{n}(\%)$ & $50(38,8)$ & $4(6,8)$ \\
Function n (\%) & $10(7,8)$ & $36(61)$ \\
Symptom n (\%) & $57(44,2)$ & $3(5,1)$ \\
Function + Symptom n (\%) & $12(9,3)$ & \\
\hline
\end{tabular}

Conclusions: As a result of this study, there was a difference between parent's and child's perception of the disease. While children report functional complaints, parents focus on symptomatic complaints. Exercise approaches should focus on the child's functional complaints and identify common goals with the family. Families should be educated about the importance of the complaints that the child expresses.

\section{References:}

[1] Manczak, M. Rutkowska-Sak, L. Raciborski, F. Health-related quality of life in children with juvenile idiopathic arthritis - child's and parent's point of view. Reumatologia 2016; 54(5):243-250.

[2] Consolaro, A. Negro, G. Lanni, S. et al. Toward a treat-to-target approach in the management of juvenile idiopathic arthritis. Clin Exp Rheumatol 2012; 30(4 Suppl 73): 157-162.

Acknowledgements: The author declare that they have no conflict of interest.

Disclosure of Interest: None declared

DOI: 10.1136/annrheumdis-2017-eular.5749

\section{SAT0731-HPR SYMPTOMS OF PAIN, FATIGUE AND SELF-EFFICACY IN YOUNG PATIENTS WITH SPONDYLOARTHRITIS - A COMPARISON BETWEEN WOMEN AND MEN}

A. Torell ${ }^{1}$, A. Bremander ${ }^{2,3,4}$, S. Bergman $3,4,5$, E. Haglund ${ }^{2,4} \cdot{ }^{1}$ Department of physiotherapy, Ängelholms sjukhus, Ängelholm; ${ }^{2}$ School of Business, Engineering and Science, Halmstad Univeristy, Halmstad; ${ }^{3}$ Department of clinical Sciences, Lund, Section of rheumatology, Lund University, Lund; ${ }^{4}$ Spenshult R\&D center, Sweden, Halmstad; ${ }^{5}$ Primary Health Care Unit, Department of Public Health and Community Medicine, Institute of Medicine, The Sahlgrenska Academy, University of Gothenburg, Gothenburg, Sweden

Background: Spondyloarthritis ( $\mathrm{SpA}$ ) often has an early disease onset with inflammatory back pain debuting already in young adulthood. Studies have shown gender differences in disease specific areas but few studies have focused only on the younger subjects. Extended knowledge based on self-reported information can help to better understand the characteristics of these younger women and men with SpA.

Objectives: To study the differences between young women and men with SpA with regard to self-reported pain measures, disease activity, fatigue, self-efficacy and health status.

Methods: A cross-sectional population based cohort of 201 patients age 1836 years with $\mathrm{SpA}$ identified through a health care register by searching for ICD-10 codes for SpA between the years 2003-2007. They all responded to a questionnaire survey in 2009. $29 \%$ were diagnosed with ankylosing spondylitis, $39 \%$ with psoriatic arthritis, and $32 \%$ with undifferentiated spondyloarthritis. The survey included questions concerning pain (NRS 0-10 and a pain mannequin), fatigue (NRS 0-10), self-efficacy (ASES 10-100, low-high). The pain mannequin was used to categorize patients into groups; no chronic pain (NCP), chronic regional pain (CRP)or chronic widespread pain (CWP). Self-reported disease activity (BASDAI 0-10) and health status (EQ5D, 0-1) were used to describe the group. Characteristic symptoms are reported as mean, standard deviation (SD) and frequencies. T-test and Chi2 test were used to study gender differences.

Results: The mean age (SD) was, $30(5)$ years, $60 \%$ were women. The group reported disease activity scores (BASDAl) of $3.8(2.3)$, health status $0.75(0.16)$, and a disease duration of 7 (5) years. One third were smokers or former smokers, and $69 \%$ reached WHO's recommended level of health enhancing physical activity. $21 \%$ reported CRP, $41 \%$ CWP and the remaining $38 \%$ reported NCP. More women reported CWP pain than men, ( $48 \%$ vs. $30 \%, p=0.026)$. Women also reported worse pain compared to men, (3.9 (2.4) vs. 2.9 (2.1), $p=0.001$ ), worse fatigue (5.0 (2.6) vs. 3.9 (2.7), $p=0.003)$, less self-efficacy for pain (53 (20) vs. 59 (21), $p=0.040$ ) and also for symptoms (59 (19) vs. 65 (20), $p=0.038$ ).

Conclusions: A significant proportion of both women and men reported symptoms consistent with chronic widespread pain already at young age. Women reported in general worse health compared to men, including pain distribution, pain intensity and pain management. This information could be valuable for clinicians in the care of young patients with $\mathrm{SpA}$.

Disclosure of Interest: None declared

DOI: 10.1136/annrheumdis-2017-eular.4624

\section{SAT0732-HPR RESILIENCE AND POSITIVE AFFECT ARE RELATED TO THE EXPERIENCE OF FATIGUE IN PATIENTS WITH A RHEUMATIC DISEASE}

E. Taal ${ }^{1}$, K. Schreurs ${ }^{1}$, L. Guddorf ${ }^{1}$, C. Bode ${ }^{1}$, M. van de Laar ${ }^{2} .{ }^{1}$ Psychology, Health \& Technology, University Twente; ${ }^{2}$ Rheumatology, Medisch Spectrum Twente, Enschede, Netherlands

Background: Fatigue is a common symptom in patients with a rheumatic disease. Resilience, the ability to bounce back or recover from stress, has been found to be related to lower fatigue in patients with cancer, traumatic brain injury, cardiac disease and fibromyalgia (see e.g. [1])

Objectives: To study the relationships of resilience and the resilience related factors positive affect, acceptance and engaged living with fatigue in patients with rheumatic diseases.

Methods: 57 patients with a rheumatic disease (rheumatoid arthritis, 70\%; osteoarthritis, $11 \%$; others, $9 \%$ ) completed an online questionnaire. Fatigue was assessed with SF-36 vitality scale; pain with a VAS; Resilience with BRS and resilience related factors with PANAS (positive affect), AAQ-II (Acceptance) and ELS (engaged living). Data were analysed with hierarchical multiple regression analyses.

Results: Resilience, positive affect, acceptance and engaged living were multivariate significantly related to fatigue $\left(R^{2}=0.54 ; P \leq 0.001\right)$. Resilience $(\beta=0.29$; $\mathrm{P} \leq 0.05)$ and positive affect $(\beta=0.39 ; \mathrm{P} \leq 0.01)$ were significant individual predictors of lower fatigue in multiple regression analysis. Acceptance and engaged living were not significantly related with fatigue in the multivariate model. The relationship between resilience and fatigue was partially mediated by positive affect. When pain was included in the model the relations of resilience $(\beta=0.27$; $\mathrm{P} \leq 0.05)$ and positive affect $(\beta=0.34 ; \mathrm{P} \leq 0.05)$ with fatigue remained significant.

Conclusions: Resilience and positive affect may be predictors of decreased fatigue in rheumatic patients. Further longitudinal studies are needed to examine the causality of these relationships.

\section{References:}

[1] Smith BW, Dalen J, Wiggins K, Tooley E, Christopher P, Bernard J. The brief resilience scale: Assessing the ability to bounce back. Int J Behav Med 2008:15(3):194-200.

Disclosure of Interest: None declared

DOI: 10.1136/annrheumdis-2017-eular.6378

\section{SAT0733-HPR A QUALITATIVE STUDY ON OBSTACLES AND MOTIVATIONS TO VACCINATIONS IN RHEUMATOID ARTHRITIS}

F. Fayet, C. Savel, M. Rodere, M. Soubrier, S. Mathieu. Rheumatology department, CHU Clermont-Ferrand, Clermont-Ferrand, France

Background: Rheumatoid arthritis (RA) is characterized by an increased risk of infection, which is further enhanced by the associated treatments like corticosteroid therapy or biologicals. To diminish this risk, influenza and 
pneumococcal vaccinations have been recommended prior to initiating treatment with biological agents. The COMEDRA trial, conducted in France, revealed the usefulness of a nurse-led consultation in the management of RA-associated comorbidities. Despite this, however, only $40 \%$ of patients were shown to be appropriately vaccinated (1).

Objectives: This study sought to better understand the obstacles encountered in RA patients as to influenza and pneumococcal vaccinations, along with their motivations.

Methods: This qualitative study was conducted at the rheumatology department by means of semi-directed nurse-led interviews lasting about 45 minutes, between July and November 2015. Four topics were analyzed: obstacles and motivations to influenza vaccination, obstacles and motivations to pneumococcal vaccination, post-vaccination follow-up, and information sources. The interviews were registered and transcribed within 45 hours post-interview, and were pursued until data saturation. Transversal data analysis was carried out by the nurse in charge of the interview, then by a second nurse, with the two analyses assembled according to the traditional concept of double reading. Differences between both analyses were thoroughly discussed, with a consensus sought by a third researcher, namely a rheumatologist, as necessary.

Results: Overall, 15 interviews were conducted involving 11 women and 4 men, with a mean age of 63 years (29-83). All patients were suffering from RA and undergoing at least one immunosuppressant therapy. Most $(80 \%)$ were vaccinated against pneumococcus, but only $33 \%$ against influenza, with eight patients declaring having been affected by influenza and one by pneumococcusrelated pulmonary disease.

The obstacles to vaccinations, revealed during the interviews, primarily concerned fears of unwanted effects in relation with the vaccinations, particularly concerning the influenza vaccination. The patients also reported anxieties in relation with the vaccines' excipients, with overdoses, or with disease reactivation. Moreover, media impact, contradictory information, the fact that vaccinations were not always proposed, and the lack of traceability may also be considered obstacles against vaccinations. Influenza is often considered a benign disease, and the vaccine poorly efficacious. Patients with prior influenza were more motivated towards vaccination. The primary motivation reported by the patients, and especially as to the pneumococcal vaccination, was the protection provided by the vaccine. The perception of frailty and increased infection risk associated with the disease and its treatments were other sources of motivation, and particularly when vaccination was highly recommended by the physicians, and when there had been no public controversy.

Conclusions: The fears and motivations associated with these two vaccinations are not identical. Influenza vaccination appears to be poorer perceived, patients reporting more fears related to it. With respect to pneumococcus vaccination, we only collected a few comments, given that the vaccine is less well know, and thus better accepted.

References:

[1] Gossec L. Arthritis Rheumatol 2016; 68 (suppl 10).

Disclosure of Interest: None declared

DOI: 10.1136/annrheumdis-2017-eular.4994

\section{SAT0734-HPR THE EFFECT OF RHEUMATIC DISEASES ON WORK ABILITY: A STUDY OF SELF-ASSESSMENT OF SF-36 IN PATIENTS WITH SMART SYSTEM OF DISEASE MANAGEMENT (SSDM)}

H. Song ${ }^{1}$, J. Gu ${ }^{2}$, H. Wu ${ }^{3}$, S. Li ${ }^{4}$, Y. Zhao ${ }^{5}$, H. Sun ${ }^{6}$, J. Huang ${ }^{7}$, X. Li ${ }^{8}$, R. Wu ${ }^{9}$, B. Wu ${ }^{10}$, C. Xiao $^{11}$, L. Qian ${ }^{12}$, F. Xiao ${ }^{13}$, H. Xiao ${ }^{13}$, M. Wang ${ }^{13}$ Y. Jia ${ }^{13}$, Z. Zhang ${ }^{5} .{ }^{1}$ Department of Rheumatology and Immunology, Department of Rheumatology and Immunology, Beijing; ${ }^{2}$ The Third Affiliated Hospital of Sun Yat-sen University, Guangzhou: ${ }^{3}$ Department of Rheumatology and Immunology, Dongguan People's Hospital, Dongguan; ${ }^{4}$ The First Affiliated Hospital of PLA General Hospital, Beijing; ${ }^{5}$ Department of Rheumatology and Immunology, The First Affiliated Hospital of Harbin Medical University, Harbin ${ }^{6}$ Shandong Provincial Hospital, Jinan; ${ }^{7}$ The sixth Affiliated Hospital of Sun Yat-sen University, Guangzhou: ${ }^{8}$ Anhui Medical University Affiliated Provincial Hospital, Hefei; ${ }^{9}$ The First Affiliated Hospital of Nanchang University, Nanchang, ${ }^{10}$ The First People's Hospital of Jingzhou, Jingzhou; ${ }^{11}$ TCM-Integrated Hospital of Southern Medical University, Guangzhou; ${ }^{12}$ The Second Affiliated Hospital of Medical University of Anhui, Hefei; ${ }^{13}$ Medical Department, Shanghai Gothic Internet Technology Co., Ltd., Shanghai, China

Background: A variety of rheumatic diseases can significantly affect the patient's quality of life and work ability. SF-36 is a commonly used tool to assess the quality of life and work ability in patients with chronic disease. In the past, most patients were guided by doctors/nurses to assess SF-36 in a paper form. At present, there is a lack of research on the quality of life in patients with rheumatic diseases by using new mobile tools in the real world.

Objectives: To explore the effect to the life ability of rheumatic disease and the potential association between the disease activity and SF-36 in patients with rheumatoid arthritis (RA) using SSDM.

Methods: SSDM is a new smart disease management mobile tool, which includes physicians' and patients' application system. After entering the results of SF-36 assessment by patients, all data can be synchronized automatically to the mobile terminal of authorized rheumatologist. According to the scores of SF-36 $(<12.5$,
$12.5-50$ and $>50$ ), the quality of life was divided into three levels: poor, moderate and good.

Results: From June 2016 January 2017, data were extracted online from the mobile terminals of 839 adult patients (284 male and 555 female) in 62 hospitals across China. All patients performed self-assessment of SF-36 for a total of 1,065 times. The mean age was $38.12 \pm 13.87$ (18 to 81 ) years and the median disease duration was 20.60 ( 0 to 573 ) months. There are 25 kinds of rheumatic diseases involved, including RA (23\%), ankylosing spondylitis (AS, 19\%), systemic lupus erythematosus (SLE, 13\%), osteoarthritis (OA, 11\%), Sjogren syndrome (SS, 10\%), polymyositis/dermatomyositis (PM/DM, 6\%), mixed connective tissue disease (MCTD, $6 \%$ ) and others (12\%).

The 8 items of SF-36 index were averaged between 49.07 (General Health perceptions, GH) to 74.12 (Physical Functioning, PF). The overall mean score of patients with different rheumatic diseases was higher than 50 . However, there were 5 kinds of rheumatic diseases with average score of 5 items less than 50 , including: MCTD, SS, PM/DM, AS and SLE in patients with GH score: 44.24, 44.60, 47.87, 47.93 and 49.16, PM/DM in patients with the Emotional Role functioning (RE): 47.52, Physical Role functioning (RP): 44.68 and AS patients with Reported Health Transition (HT): 49.64, respectively. In RA patients, $38 \%$ and $37 \%$ of patients reported that their ability to work were affected by disease and mood changes respectively. Bivariate correlation analysis showed that DAS28 was negatively correlated with the two item scores (RP and RE) of SF-36 index in RA patients, $p<0.01$.

Conclusions: SSDM can be used to assess SF-36 in patients with rheumatic disease. The Quality of life scores in patients with MCTD, SS, PM/DM, AS, and SLE were more likely to be affected by rheumatic disease. The disease activity (DAS28) of RA patients was negatively correlated with the work ability (RP and RE of SF-36 score)

Disclosure of Interest: None declared

DOI: 10.1136/annrheumdis-2017-eular.4990

\section{SAT0735-HPR PATIENTS' ASSESSMENT OF THE APPLICABILITY OF THE ULTRASOUND IMAGE METHOD}

J.A. Mendonça ${ }^{1}$, W.H. Rodrigues Ferreira ${ }^{2}$, I.M.M. Laurindo ${ }^{3} .{ }^{1}$ Rheumatology, Pontifical Catholic University of Campinas, Campinas, ${ }^{2}$ Rheumatology, Grupari Petrópolis, Petrópolis; ${ }^{3}$ Rheumatology, Universidade Nove de Julho, São Paulo, Brazil

Background: The applicability of ultrasonography in diagnosis and monitoring of rheumatic diseases has been steadily growing but little is known about the patient's perspective.

Objectives: To assess the patient's knowledge of the ultrasound (US) method and its clinical applicability as well as to evaluate how much was learned after a lecture.

Methods: Patients with rheumatic diseases were invited to an educational meeting that included an US lecture. They were asked to fill out a questionnaire before and after the 35-minute lecture about US and its clinical uses. The questionnaire included questions about their disease and treatment, their educational background (years of study) and ten questions about US and its clinical uses (table). These ten questions had to be answered with "yes", "no", or "I am not sure".

Results: A total of 70 patients attended the lecture and answered the questionnaires. Their demographic characteristics were as follows: Mean \pm SD: age $63 \pm 12$ years, disease duration: $9.6 \pm 0.8$ years, the most frequent self- reported diagnosis was rheumatoid arthritis $(57,2 \%)$, while osteoarthritis accounted for another $20 \%$. Most patients had only eight years of study $(64.3 \%)$ and a university degree was reported by $14 \%$. The questionnaire responses of 66 patients with rheumatic diseases were analysed. There was a noticeable increase in the positive responses to these questions: 1, 2, 3 and 7 , after the lecture, $44 \%$

Table - Questions :
1)Do you know what arthritis is?
2)Do you know what an ultrasound exam is?
3) Do you believe that an ultrasound exam could help in the diagnosis of your disease?
4) Do you believe that an ultrasound exam could help in monitoring your treatment?
5)Have you ever had one of your joints examined by ultrasound?
6) Has the exam changed your treatment?
happening to your joints?
8)Do you think that an ultrasound exam would help you follow the treatment?
9) Do you believe that an ultrasound exam can detect inflammation in your joints even when
it is not clinically diagnosable by your doctor?

10)Do you think that an ultrasound exam would help you to understand your illness better? 